\title{
Complex time-delay dynamical systems of quadratic polynomials mapping
}

\author{
Yuanyuan Sun · Zhixing Lu • Peng Li
}

Received: 14 January 2014 / Accepted: 24 August 2014 / Published online: 9 September 2014

(C) The Author(s) 2014. This article is published with open access at Springerlink.com

\begin{abstract}
Time delays widely exist in the complex dynamical systems. In this paper, time-delay complex dynamical systems are studied by analyzing the properties of corresponding Julia sets. Experimental results show that the stability of complex dynamical system changes greatly due to the influence of time delay. The variations in time-delay Julia sets are studied. In addition, the conditions holding the dynamical systems stable are found. Time delay affects the stability of complex dynamical systems, and the existence of the fixed and periodic points is also discussed.
\end{abstract}

Keywords Time delay - Complex dynamical systems $\cdot$ Julia sets $\cdot$ Fractal

\section{Introduction}

In the 1970 s, Julia sets were obtained by mathematician Gaston Julia who studied the simple iterative map in the complex plane. In recent years, there are many developments in Julia sets, including dynamical systems [1-3], complex networks [4-6], and the fractal arts [7].

As we know, dynamical system theory began with Newton in the seventeenth century and took a radical turn with Poincare a century ago. The dynami-

Y. Sun $(\varangle) \cdot$ Z. Lu $\cdot$ P. Li

College of Computer Science and Technology, DaLian

University of Technology, Dalian, China

e-mail:syuan@dlut.edu.cn cal systems occur in four different flavors [8] such as flows, cascades, iterations, and shifts. The complex dynamical systems are associated with the iteration (repeated application) of a rational mapping of the complex plane, such as Julia sets.

The existence of a delay in a system model could have several causes, for example the measure of a system variable or the physical nature of a signal transmission. Most research works on time delay were about differential dynamical systems [9-12] and neglected the problems of the complex dynamical systems. In this paper, we are concerned with the time-delay complex dynamical systems by drawing corresponding Julia sets.

The outline of this paper is as follows. We devote Sect. 2 to a discussion of governing equations and the theoretical analysis about complex time-delay dynamical systems. Definition of time-delay iteration and the numerical experiments is presented in Sect. 3, showing how the time delay affects the stability of complex dynamical systems.

\section{Governing equations and theoretical analysis}

2.1 Time-delay equation and theoretical analysis

For each quadratic polynomial $R(z)=a z^{2}+2 b z+d$, we have a polynomial $M(z)=a z+b$ and a quadratic polynomial $f(z)=z^{2}+c$, such that $M^{-1} \circ f \circ M=$ $R(z)$ [13]. It is clear that $f(z)$ is a conformal mapping 
of $R(z)$ and $f(z)$ has the same dynamical system as $R(z)$. In this case, the way to investigate the properties of complex quadratic polynomials is to study $f(z)=$ $z^{2}+c$.

We draw the time-delay Julia set utilizing the escape time algorithm [14], which is a common method to draw a fractal set. For $f(z)=z^{2}+c$, we define $f^{n}$ to be $\underbrace{f \circ f \circ \cdots \circ f}_{n}$, and $f_{n}(z)=x_{n}+i y_{n}, c=p+i q$, where $p, q, x_{n}, y_{n} \in R, n \in N_{+}$. So $\left(x_{n}, y_{n}\right)$ can be calculated by Eq. (1) as follows:

$\left\{\begin{array}{l}x_{n}=x_{n-1}^{2}-y_{n-1}^{2}+p \\ y_{n}=2 x_{n-1} y_{n-1}+q\end{array}\right.$

There are two axes of coordinates for each complex iterate function, so the time delay may happen on either of them. When a time delay happens on the abscissa axis, Eq. (1) should be changed into

$\left\{\begin{array}{l}x_{n+1}=x_{n} \\ y_{n+1}=2 x_{n} y_{n}+q\end{array}\right.$

Equation (2) can be simplified as $y_{n+1}=2 x_{n} y_{n}+q$.

Now, a complex dynamical system problem is changed into the problem about a real dynamical system. Then, we carry out the following theorem:

Theorem 1 Let $(x, y)$ be a point under the iteration of Eq. (2), (x,y) can hold on being stable if and only if the absolute value of $x$ is less than or equal to half.

Proof According to mathematical induction, the result of $(x, y)$ iterated on Eq. (2) is as follows:

$$
\begin{aligned}
& 2 x y+q,(2 x)^{2} y+q(2 x+1), \ldots,(2 x)^{n} y \\
& \quad+\left[(2 x)^{n-1}+\cdots+2 x+1\right] q .
\end{aligned}
$$

Let $y_{n}$ be the value of $(2 x)^{n} y+\left[(2 x)^{n-1}+\cdots+\right.$ $2 x+1] q$; we can get the result of $y_{n}$ after $n$ iterations by the property of geometric sequence,

$$
y_{n}=(2 x)^{n-1}\left(2 x y-\frac{q}{1-x}\right)+\frac{q}{1-x} .
$$

It is easy to see that

$$
\lim _{n \rightarrow+\infty} y_{n}=Y=\left\{\begin{array}{cc}
\frac{q}{1-x} & |x|<1 / 2 \\
2 x y & |x|=1 / 2 \\
+\infty & |x|>1 / 2
\end{array}\right.
$$

If the absolute value of $x$ is less than or equal to half, the result is convergent.

The proof is completed.
When time delay happens on the ordinate axis, Eq. (1) should be changed into

$$
\left\{\begin{array}{l}
x_{n+1}=x_{n}^{2}-y_{n}^{2}+p \\
y_{n+1}=y_{n}
\end{array}\right.
$$

We study the iterate function $f(x)=x^{2}+t$, where $t=p-y_{n}^{2}, x_{n}=x$. Then, we can carry out Theorem 2 .

Theorem 2 For iterate function $f(x)=x^{2}+t$, if $t>$ $\frac{1}{4}, f(x)$ does not have fixed point and period-2 points; if $t \leq \frac{1}{4}, f(x)$ has two fixed points; if $t \leq-\frac{3}{4}, f(x)$ has two period-2 points.

Proof Let $x^{2}+t=x$; it is clear that if $t$ is greater than one-quarter, there is no solution for the equation, so there is no fixed point for the iterate function $f(x)$. If $t$ is less than or equal to a quarter, the fixed points are $\frac{1}{2} \pm \sqrt{\frac{1}{4}-t}$.

Let $\left(x^{2}+t\right)^{2}+t=x$; the equation can be changed as $\left(x^{2}-x+t\right)\left(x^{2}+x+1+t\right)=0$. It is easy to know that if $t>-\frac{3}{4}$, there is no period-2 points in the dynamical systems; if $t \leq-\frac{3}{4}$, there are two period-2 points,

$$
\begin{aligned}
& x_{1}=-\frac{1}{2}+\sqrt{-\frac{3}{4}-t} \text { and } x_{2}=-\frac{1}{2}-\sqrt{-\frac{3}{4}-t} . \\
& \text { The proof is completed. }
\end{aligned}
$$

In order to analyze properties of the period-2 points, let

$q_{c}^{ \pm}=-\frac{1}{2} \pm \sqrt{-\frac{3}{4}-t}$,

Then, we can get

$$
\begin{aligned}
& \left|\left(f_{t}^{2}\right)^{\prime}\left(q_{t}^{ \pm}\right)\right|=\left|f_{t}^{\prime}\left(q_{t}^{-}\right)\right| \cdot\left|f_{t}^{\prime}\left(q_{t}^{+}\right)\right| \\
& \quad=\left|2 \times\left(-\frac{1}{2}-\sqrt{-\frac{3}{4}-t}\right)\right| \cdot\left|2 \times\left(-\frac{1}{2}+\sqrt{-\frac{3}{4}-t}\right)\right| \\
& \quad=4|1+t|
\end{aligned}
$$

As we know, for an iterative function $f\left(q_{c}^{ \pm}\right)$, if

$$
\mid \begin{aligned}
& f\left(q_{c}^{ \pm}\right)^{\prime} \mid<1, \quad q_{c}^{ \pm} \text {are attractive, } \\
& \left|f\left(q_{c}^{ \pm}\right)^{\prime}\right|>1, \quad q_{c}^{ \pm} \text {are repelling, } \\
& \left|f\left(q_{c}^{ \pm}\right)^{\prime}\right|=1, \quad q_{c}^{ \pm} \text {are neuter }[15] .
\end{aligned}
$$

According to Theorem 2 and [15], we can get a consequence as:

Consequence 1 If $-\frac{5}{4}<t<-\frac{3}{4}$, the periods are attractive;

if $t<-\frac{5}{4}$, the periods are repelling; 
if $t=-\frac{3}{4}$ or $t=-\frac{5}{4}$, the periods are neuter.

The proof is easy to be obtained.

For short-lived time-delay Julia sets, if the points are in Julia sets after time-delay iterations, they will converge to the periodic points finally. Their initial points are in the Julia sets. On the contrary, if the points are out of Julia sets after time-delay iterations, they will escape in the end. Therefore, their initial points are not in the Julia sets.

For the sustained time-delay Julia sets, the abscissa axis and ordinate axis time delays are considered separately. When the time delay happens on the ordinate axis, the convergence of the iterative system is determined by the absolute value of abscissa, which is the initial value of time-delay iteration. If it is not greater than half, the iterative system is convergent, otherwise it is divergent. When the time delay happens on the abscissa axis, we should analyze the properties of the iterative system periodic points.

\section{Algorithms design and numerical experiments}

\subsection{Time-delay iteration}

Because the time delay may happen on all the iterations, without the loss of generality, we suppose it happens when the dynamical system is in initial, unstable, and stable statuses. Let $k$ is the time of the iteration, $n$ is the time when the time delay occurs. In our experiments, the time limit of the escape time algorithm is 300 . The $k$ is a loop variable of the algorithm; therefore, the range of $k$ is $[1,300]$. According to the experimental results, almost all the convergent initial points in complex plane will converge into Julia sets after 200 iterations, so we consider the dynamical system is stable at this moment. It is regarded as an unstable status when the graph is similar with a Julia set and only a part of it is fractal. The experimental results show that when $n$ is in [15, 30], most of the dynamical systems are unstable. For the Julia set of $c=-0.08+0.68 i$ or $c=0.305+$ $0.5475 i$, the range of $n$ is $[20,25]$. In our experiments, the dynamical system is unstable when $n=20$ and is initial when $n=1$. For each status, we design the iteration as follows:

(1) Short-lived time delay

$$
\begin{aligned}
& k<n, \text { using Eq. (1) to calculate the value of } \\
& \left(x_{n}, y_{n}\right) . \\
& n \leq k<n+5 \text {, using Eqs. (2) or (3). } \\
& n \geq n+5 \text {, using Eq. (1). }
\end{aligned}
$$

(2) Sustained time delay

$$
\begin{aligned}
& k<n \text {, using Eq. (1) to calculate the value of } \\
& \left(x_{n}, y_{n}\right) \text {. } \\
& k \geq n \text {, using Eqs. (2) or (3). }
\end{aligned}
$$

\subsection{Numerical experiments and analysis}

For $f(z)=z^{2}+c$, let $c=-0.08+0.68 i$ or $c=0.305+0.5475 i, R$ is the escape radius, and $R \geq \max (2,|c|)$. Figure 1 shows no time-delay Julia sets. There are a short-lived and a sustained time-delay Julia set on each couple of graphs (as shown in Figs. 2, $3,4,5)$. For short-lived time delay, the duration of the
Fig. 1 No time-delay Julia sets a $c=-0.08+0.68 i$. b $c=0.305+0.5475 i$
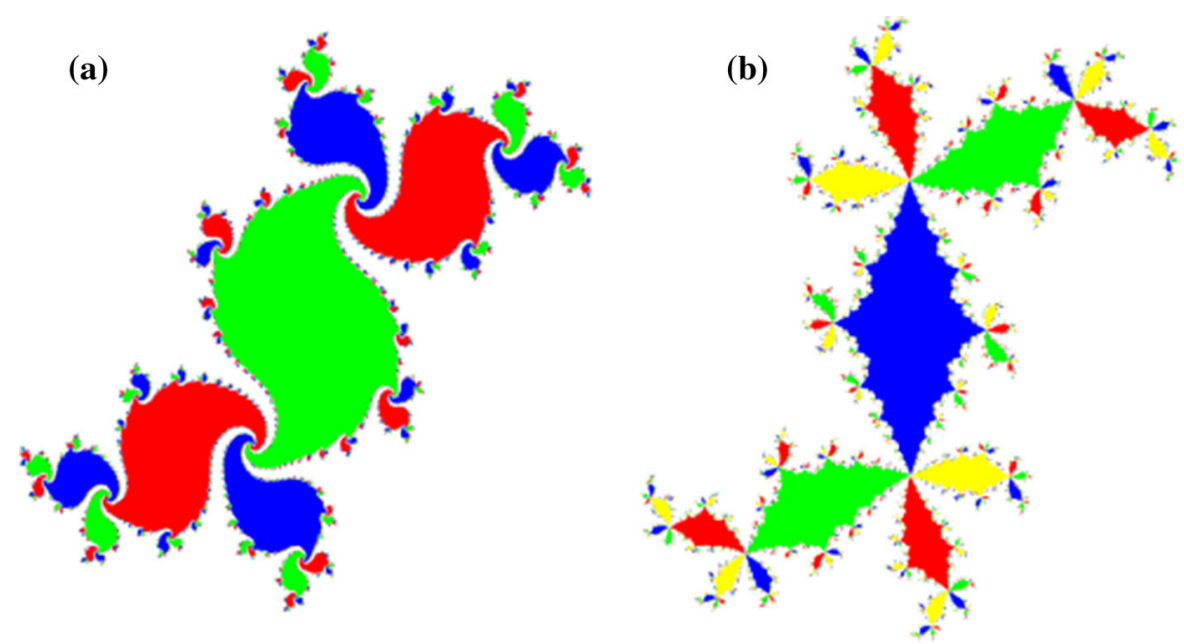
Fig. 2 Julia sets of time-delay iterations which happen on the initial status $(n=1)$ (SLTD short-lived time delay, STTD sustained time delay). a SLTD on ordinate axis. b STTD on ordinate axis. c SLTD on abscissa axis. $\mathbf{d}$ STTD on abscissa axis

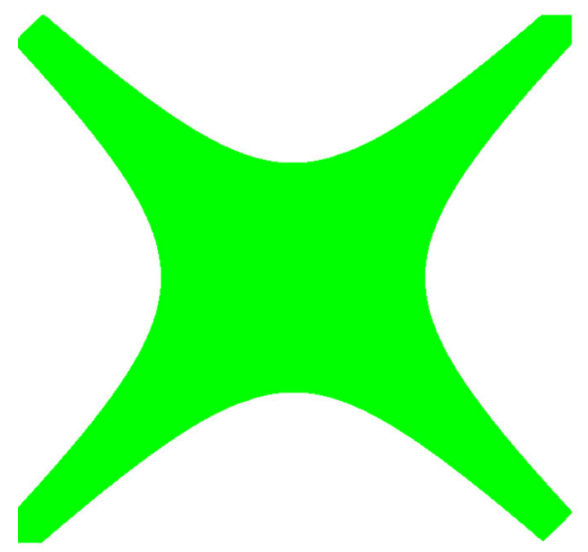

(a)

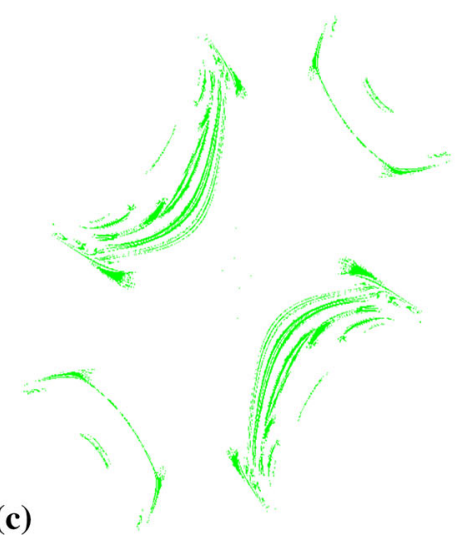

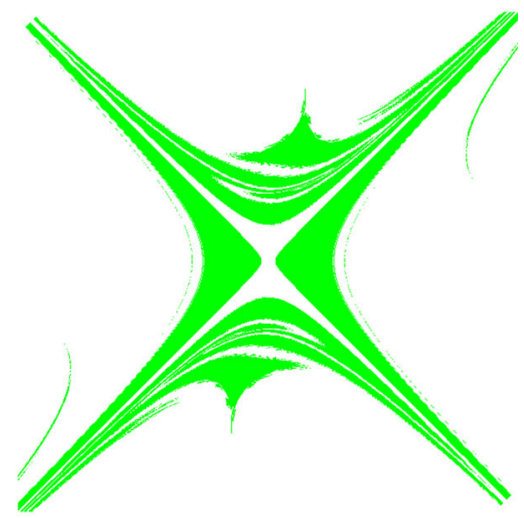

(b)

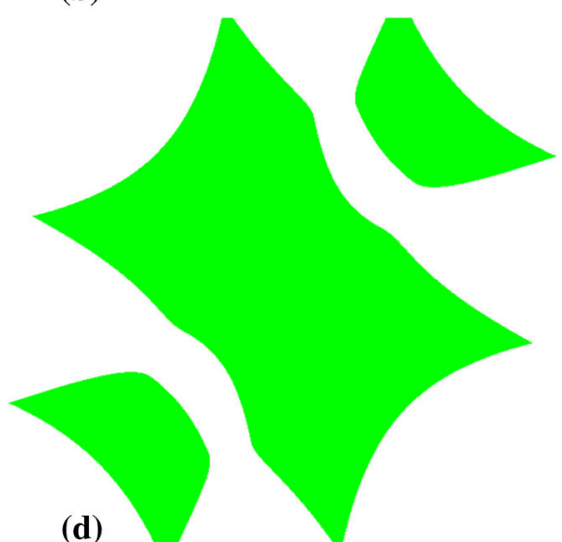

time delay is five because most escaped points will go out of the Julia set after five iterations. However, if the delay time is too short, most of the points will be still in the Julia set and the time delay may have little impact on the Julia set.

Figure 2 shows the Julia sets of time-delay iterations which happen on the initial status. Note that they are not fractals. Figure 2a, b is about ordinate axis timedelay Julia sets; Fig. $2 \mathrm{a}$ is the Julia set with short-lived time-delay iterations; Fig. $2 \mathrm{~b}$ is the sustained timedelay Julia set. Figure $2 c$, $d$ is the abscissa axis timedelay Julia sets. When the time-delay iteration happens, the complex dynamical system changes into a real dynamical system and is greatly destroyed by the initial time-delay iterations. For the short-lived time delay, although it only sustains for five iterations at initial status of the dynamical system, it leads to a largely impact.

Figure 3 shows the Julia sets when time-delay iterations happen on the twentieth iteration. The results are similar to the traditional Julia sets. Some points in the
Julia sets escape periodically, because only a part of initial points converge to the periods after twenty iterations. Each part of the Julia set which is escaped after time-delay iterations is connected. There is no point which is escaped but whose any neighborhood point is convergent in the Julia sets. This is due to the fact that the points in Julia sets are dense. Dense point sets are still dense under one-dimensional real dynamical systems. There is no isolated point in time-delay Julia sets. Time-delay iteration destroys the fractal feature of the Julia set but does not change its density.

Figures 4 and 5 show the time-delay Julia sets at stable status on the ordinate and abscissa axis, respectively. The Julia sets in these pictures are fractals. Only periodic points are affected by the time-delay iteration after the dynamical system is stable. If a periodic point in the Julia set is escaped under the time-delay iteration, then all the initial points converging to the periodic point will escape.

For the abscissa axis time-delay Julia sets, according to Theorem 1 , if the abscissa absolute value of a 
Fig. 3 Julia sets of time-delay iterations which happen on unstable status $(n=20)$. a SLTD on ordinate axis. b STTD on ordinate axis. c SLTD on abscissa axis. $\mathbf{d}$ STTD on abscissa axis
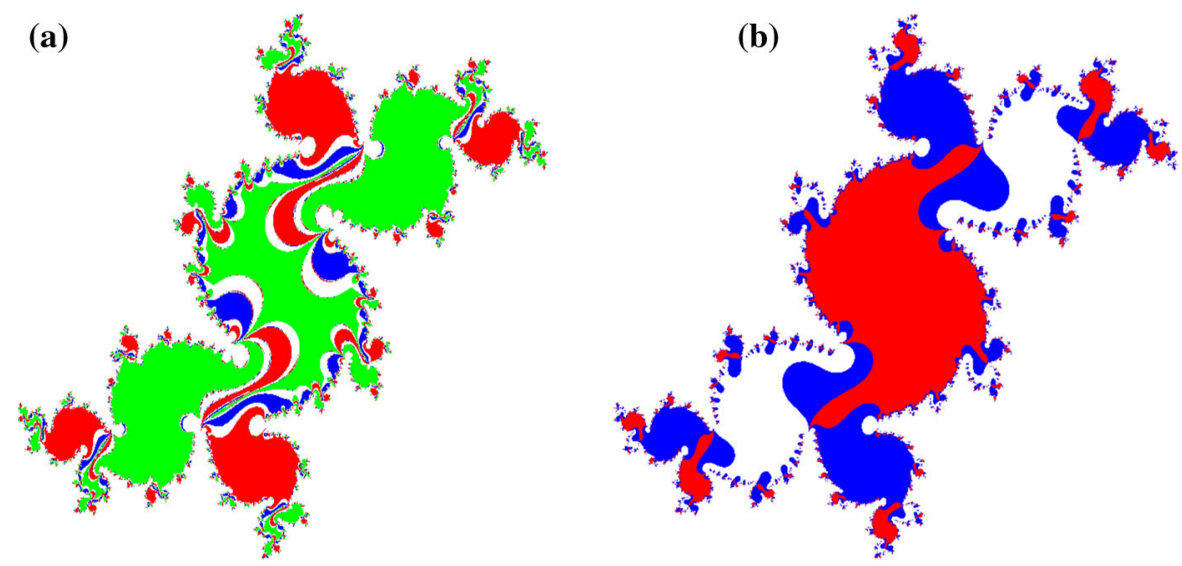

(c)

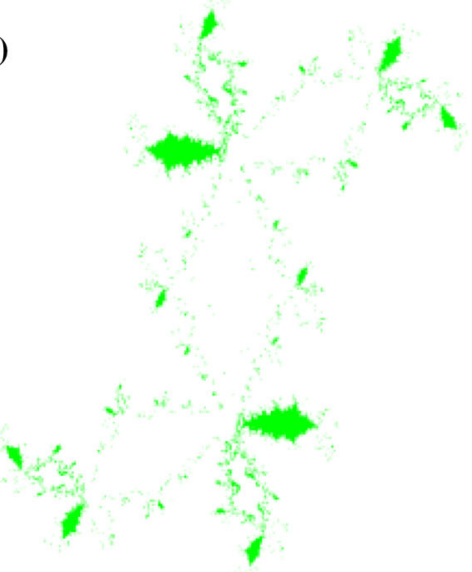

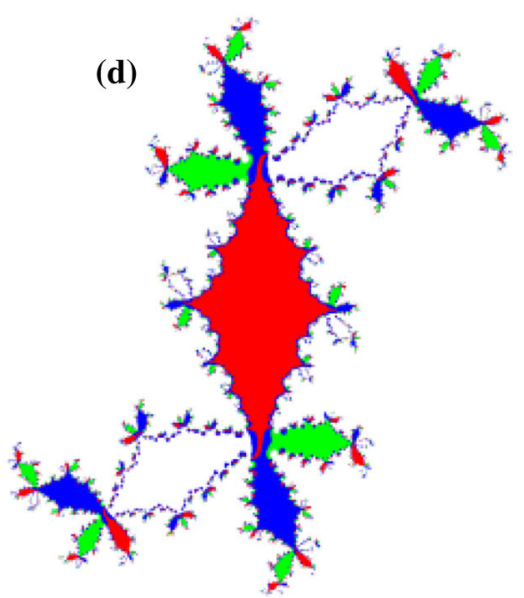

point is less than or equal to half, then the point will be convergent. When $c=-0.08+0.68 i$, the periodic points are

$P_{1}(-0.045858,0.668557)$,

$P_{2}(-0.524866,0.618683)$,

$P_{3}(-0.187284,0.030549)$.

The iterative functions starting with $P_{1}$ and $P_{3}$ are convergent. $P_{1}$ is much faster than $P_{3}$ when they converge to the stable points. $P_{2}$ is divergent under the sustained time-delay iteration. Because 0.524866 is near to a half, $P_{2}$ diverges slowly. In our experiment, the value of $P_{2}$ is 548,419.176875 after 300 iterations.

The time when an initial point converges to each periodic point of the Julia set is different [16]. For example, if a point converges to $P_{1}$ after 200 iterations, it will converges to $P_{2}$ after two 201 iterations, converges to $P_{3}$ after 202 iterations, and converges to $P_{1}$ after 203 iterations. Because the time-delay iterative system which starts with $P_{1}$ or $P_{3}$ is converge, the point is convergent when the time delay happens on 200th or 202th iteration, it is divergent when the time delay happens on 201th iteration. That is why the petals of the Julia set are lost by periodicity.

For the ordinate axis time delay, the convergences of the iterative functions depend on the ordinate axis values when time-delay iterations start. For the Julia set with $c=0.305+0.5475 i$, its periodic points are

$$
\begin{aligned}
& Q_{1}(-0.093552,0.87272), \\
& Q_{2}(-0.447889,0.71079), \\
& Q_{3}(0.000382,-0.089209), \\
& Q_{4}(0.297042,0.547432) .
\end{aligned}
$$

For $t_{n}=p-y_{n}^{2}, n=1,2,3,4$, then $t_{1}=$ $-0.456640, t_{2}=-0.200222, t_{3}=0.2970417, t_{4}=$ $0.005318 . t_{1}, t_{2}$, and $t_{4}$ are $<1 / 4$. According to Consequence $1, Q_{1}, Q_{2}$, and $Q_{4}$ converge to stable points. Since $t_{3}$ is $>1 / 4, Q_{3}$ is divergent. 
(a)

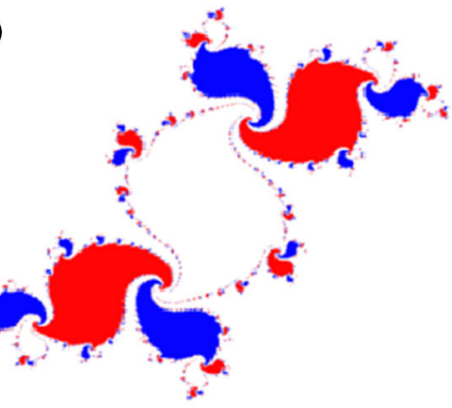

(d)

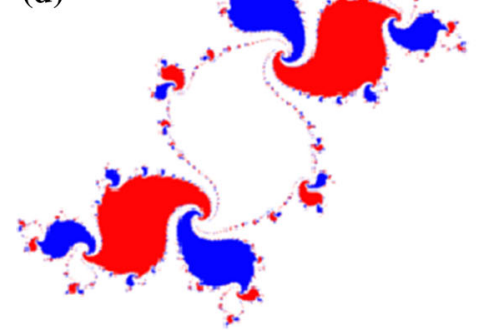

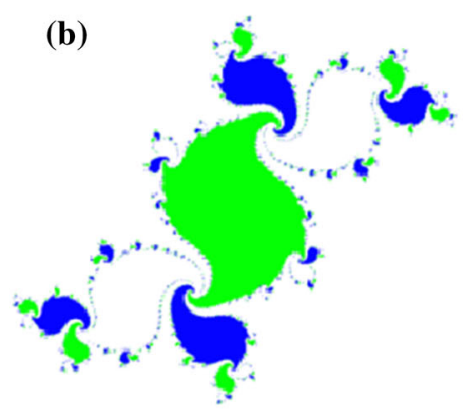

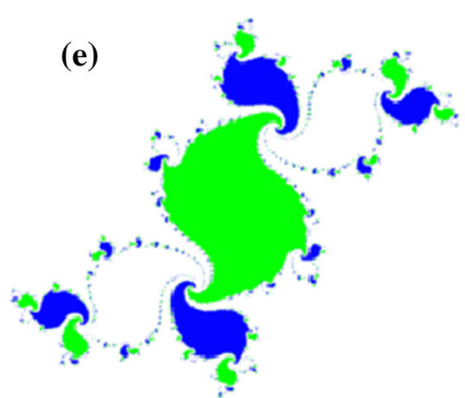

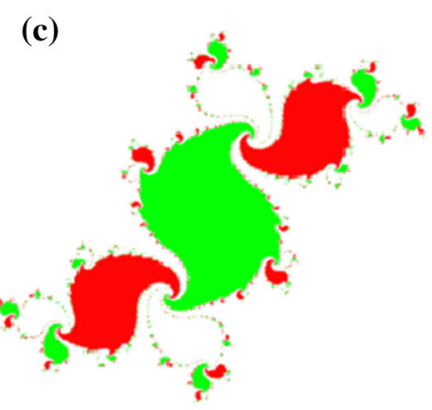

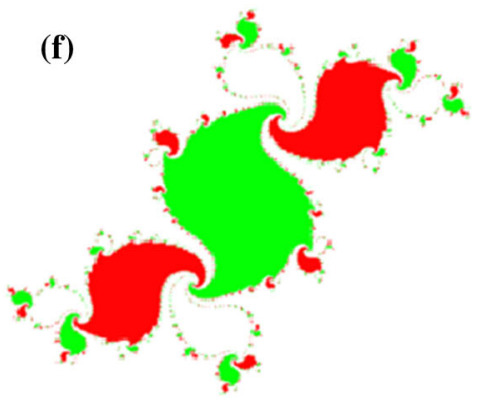

Fig. 4 Time-delay Julia sets on the ordinate axis at stable status $(n=200)$. a SLTD on 200th iteration. b SLTD on 201th iteration. $\mathbf{c}$ SLTD on 202th iteration. $\mathbf{d}$ STTD from 200th iteration. e STTD from 201th iteration. f STTD from 202th iteration

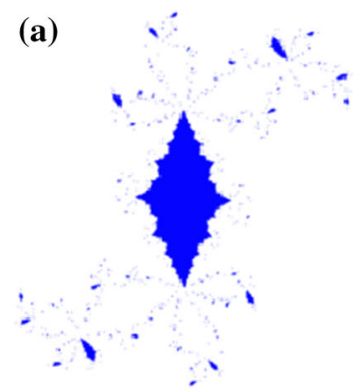

(e)

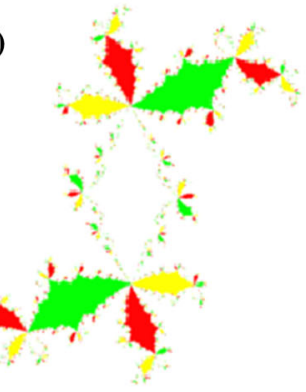

Fig. 5 Time-delay Julia sets on abscissa axis at stable status $(n=200)$. a SLTD on 200th iteration. b SLTD on 201th iteration. c SLTD on 202th iteration. d SLTD on 203th iteration.
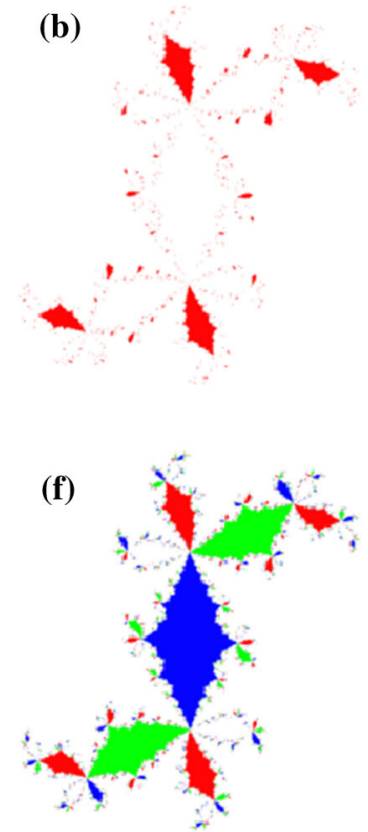

(c)

(d)
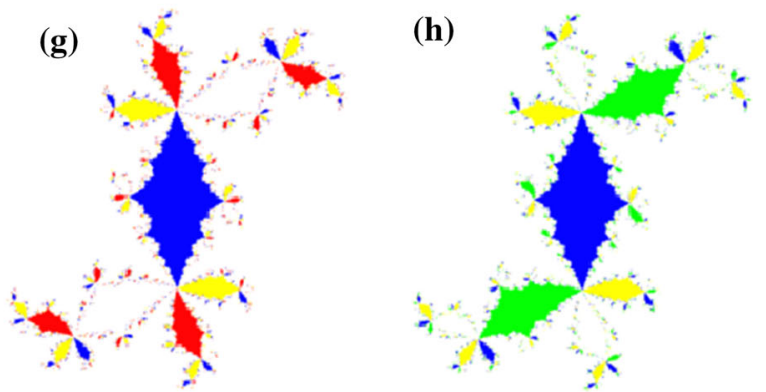

e STTD from 200th iteration. f STTD from 201th iteration. $\mathbf{g}$ STTD from 202th iteration. h STTD from 203th iteration 
There is an interesting phenomenon, the lost petals change clockwise. It is because that the periodic points change counterclockwise. Our experiments have proved that the argument of each periodic point becomes larger under the complex iteration. So $Q_{3}$ follows by $Q_{2}$, does not follow by $Q_{1}$.

\section{Conclusions}

In the foregoing numerical analysis, time-delay Julia set is discussed. Time-delay iterations can destroy fractal feature of Julia sets when it happens on initial and unstable status. In some cases, the branches of the timedelay Julia sets may disappear in turn, which also shows some kind of periodicity.

For the abscissa axis time delay, the system can maintain stability if and only if the absolute value of abscissa is less than or equal to half.

For the ordinate axis time delay, if $p-y_{n}^{2}$ is on $\left(-\frac{5}{4}, \frac{3}{4}\right)$, points are attractive; if $p-y_{n}^{2}=-\frac{3}{4}$ or $p-$ $y_{n}^{2}=-\frac{5}{4}$ periods are neuter; if $p-y_{n}^{2}$ is on $\left(-\infty,-\frac{5}{4}\right)$ and $\left(-\frac{3}{4},+\infty\right)$, periods are repelling.

Although the time-delay iteration in this study is based on the mapping of a quadratic polynomial, the research methods can also be applied to other mappings, such as high-order polynomials. Not only does our study shed light on an example for the studies of the complex time-delay dynamical systems, it also provides a template for the analysis of this type of dynamical systems. Future work will focus on the mappings of general polynomials and more complex time-delay conditions.

Acknowledgments This research is supported by the National Natural Science Foundation of China (No. 61103147, 61075018, 61070098), the National Key Project of Science and Technology of China (No. 2011ZX05039-003-4), and the Fundamental Research Funds for the Central Universities (No. DUT12JB06).

Open Access This article is distributed under the terms of the Creative Commons Attribution License which permits any use, distribution, and reproduction in any medium, provided the original author(s) and the source are credited.

\section{References}

1. Ye, R., Zhao, H.: Control of 3D Julia sets generated by commutative hypercomplex dynamical systems. Adv. Comput. Sci. Appl. 1(1), 21-25 (2012)

2. Zhang, Y., Guo, X.: Control of Julia sets of the complex Henon system. Nonlinear Dyn. 69(1-2), 185-192 (2012)

3. Qiao, H., Kan, X., Duan, J. : Escape probability for stochastic dynamical systems with jumps. In: Malliavin Calculus and Stochastic Analysis, pp. 195-216. Springer, US (2013)

4. Song, C., Havlin, S., Makse, H.A.: Self-similarity of complex networks. Nature 433(7024), 392-395 (2005)

5. Zheng, S., Shao, W.: Mixed outer synchronization of dynamical networks with nonidentical nodes and output coupling. Nonlinear Dyn. 73(4), 2343-2352 (2013)

6. Seifi, M., Junier, I., Rouquier, J.B., Iskrov, S., Guillaume, J.L.: Stable community cores in complex networks. In: Complex Networks, pp. 87-98. Springer, Berlin (2013)

7. Guliciuc, V.: Fractal art as genuine art. In: Proceedings of the XXII World Congress of Philosophy, pp. 93-102 (2012)

8. Liu, B., Lu, W., Chen, T.: Stability analysis of some delay differential inequalities with small time delays and its applications. Neural Netw. 33, 1-6 (2012)

9. Abraham, R.: Complex Dynamical Systems. Aerial Press, Ghent (1990)

10. Kablar, N., Kvrgi, V., Debeljkovi, D.L.: Singularly impulsive dynamical systems with time delay: mathematical model and stability. Trans. FAMENA 37(3), 65-74 (2013)

11. Sun, W.: Random walks on generalized Koch networks. Phys. Scr. 88(4), 045006 (2013)

12. Sun, W., Zhang, J., Wu, Y.: Novel evolving small-world scale-free Koch networks. J. Stat. Mech. Theory Exp. (03), P03021 (2011)

13. Lu, Y.N.: Complex Analytic Dynamical Systems. Science Press, Beijing (1995)

14. Mandelbrot, B.B.: The Fractal Geometry of Nature. W.H. Freeman and Company, New York (1983)

15. Robinson, R.C.: An Introduction to Dynamical Systems. China Machine Press, Beijing (2006)

16. Sun, Y.Y., Zhao, X.D., Hou, K.N.: Calculation of Julia set by equipotential point algorithm. Int. J. Bifurc. Chaos. 23(1), 1350015 (2013) 